\title{
SECADO ARTIFICIAL DE MADERA DE TEPA (Laurelia philippiana \\ Looser)
}

\author{
Miguel Peredo L. (*) \\ Jorge Uarac S. (*)
}

\section{RESUMEN}

Se aplica un programa de secado convencional para madera aserrada de tepa (Laurelia philippiana Looser) de $1 \times 6-10^{\prime \prime} \times 3,60 \mathrm{~m}$. Las condiciones de secado se evalúan en función de la calidad final de las piezas muestreadas.

La madera utilizada en este estudio proviene de predios ubicados en la $X$ Región y fue proporcionada por la empresa EMASIL S.A.

El tiempo de secado empleado es de 96 horas para disminuir el contenido de humedad de $53,47 \%$ hasta $12,93 \%$, lo que permite calificar el programa utilizado como satisfactorio.

Los defectos que presentan mayor participación son los alabeos, seguidos de las grietas. La encorvadura es el alabeo que se presenta en mayor proporción en las piezas muestreadas, correspondiendo en su gran mayoría a piezas con corte mixto. El alabeo de segunda importancia es la acanaladura, que se presenta en el mayor número de piezas.La arqueadura y torcedura se presentan en menor proporción en las piezas muestreadas.

Los valores promedio de contracción radial y tangencial experimentados por las piezas son 3,34 y $4,70 \%$, respectivamente.

Palabras Claves : Maderas nativas; Tepa; Secado Artificial.

(*) Ingeniero Forestal, Universidad Austral de Chile, Casilla 567, Valdivia

(*) Ingeniero Forestal, Forestal Puerto Montt, Miramar 1.500, Puerto Montt. 


\section{ABSTRACT}

A conventional drying program for sawnwood of Tepa, Laurelia philippiana Looser, cut to $1 \times 6$ to $10^{\prime \prime} \times 3.6 \mathrm{~m}$ size, was elaborated. Drying conditions were evaluated according to final wood quality.

The wood under study was provided by EMASIL S.A., a company located in the $X$ th region.

It took 96 hours to lower the moisture content from $53,47 \%$ to $12,93 \%$ which allows to consider the drying programme as satisfactory. The most frecuent problems encountered after the drying process consisted in warps and cracks. Crookedness was the warp that showed the highest participation in the sample lot of sawn pieces, mainly in pieces with mixed cutting. Cupping appeared to be the warp of second importance. Bows and twists appeared in less proportion in the sample pieces.

The average tangential contraction was higher than the average radial contraction with $4,70 \%$ and $3,34 \%$ respectively.

Key words: Native wood, Tepa, Kin drying. 


\section{INTRODUCCION}

El desarrollo del Sector Forestal en los últimos años ha exigido mejores y más eficientes tecnológicas en las empresas del rubro, con el fin de obtener productos de mejor calidad y más acabados, acrecentar el prestigio de la madera chilena en el exterior y aumentar su valor agregado.

Es por esto que en el país uno de los tipos de tecnología que ha captado un creciente interés ha sido el proceso de secado de la madera, cuya importancia radica fundamentalmente en el hecho que permite lograr su estabilización dimensional, un elaborado de mejor calidad, aumentar su resistencia al deterioro por agentes bióticos, disminuir su peso y mejorar las propiedades mecánicas, entre otras razones.

En Chile se han realizado estudios de procesos de secado artificial convencional, los que en su gran mayoría se han orientado a especies exóticas, existiendo de esta manera una escasa información para las especies nativas. Esto se debe en cierta forma a que este recurso pocas veces ha sido utilizado con una visión de mejoramiento.

Entre las especies nativas con mejores proyecciones desde el punto de vista comercial se encuentra la tepa (Laurelia philippiana Looser), cuya utilización en la industria del sector ha sido significativa y es en la actualidad una de las especies más abundantes de los bosques nativos chilenos (CONAF, 1986).

Su utilización abarca diferentes productos, tales como madera aserrada y elaborada, batientes para puertas, tablas para pisos, forros y traslapos, etc. Por este motivo existe un gran interés dentro de las industrias del sector por contar con información respecto de sus caracteristicas tecnológicas con el fin de lograr mejores calidades en el producto final a obtener, calidad en la cual el proceso de secado juega un rol trascendental.

El objetivo central del presente trabajo es evaluar y analizar los resultados obtenidos con un programa de secado convencional propuesto para la Laurelia philippiana. 


\section{MATERIAL Y METODO}

\section{Material}

Madera

Las piezas de madera que se utilizaron en este ensayo se obtuvieron de trozas procedentes de bosques ubicados en la X Región.

Las características de esta madera son las siguientes:

- Escuadria: 1" de espesor

$6^{\prime \prime}$ a $10^{\prime \prime}$ de ancho

$3,6 \mathrm{~m}$ de largo

Equipo de Secado

Cámara de secado convencional ubicada en la Planta $N^{\circ} 2$ de la empresa EMASIL S.A., Valdivia, cuya capacidad aproximada es de 600 pulgadas madereras.

El movimiento del aire dentro de la cámara se logra a través de un ventilador ubicado en la parte superior de la cámara, cuya fuente de poder es un motor de 4,4 kVA de potencia y $940 \mathrm{rpm}$. Este ventilador cuenta además con un partidor e inversor de marcha, lográndose con esto modificar la dirección del aire dentro de la cámara.

La temperatura se logra mediante vapor generado por una caldera alimentada con desechos de madera producidos por la misma empresa. 


\section{Método}

El estudio realizado se lleva a cabo en condiciones de trabajo habituales en la empresa, lo que permite no alterar mayormente a el flujo de producción que esa planta posee. Esto contribuye a que los resultados y análisis abarquen una perspectiva lo más cercana posible a la realidad industrial.

\section{Proceso de Secado}

El programa de secado se confeccionó sobre la base del contenido de humedad de la madera, es decir en base a la pérdida de humedad que experimenta la madera en el transcurso del secado.

Preparación de la Muestra

Dentro de esta etapa se llevó a cabo la selección de las piezas necesarias para efectuar el secado a partir de la disponibilidad de madera aserrada.

La carga que posteriormente ingresaría a la cámara de secado se ordenó en seis paquetes, cuyas dimensiones individuales correspondian a un ancho aproximado de 1,4 $\mathrm{m}$ y una altura aproximada por paquetes de $0,8 \mathrm{~m}$.

La ordenación de cada paquete se llevó a cabo utilizando separadores cepillados de $25,4 \mathrm{~mm}$ de espesor y de $1,4 \mathrm{~m}$ de largo. Cada separador se ubicó a una distancia de $0,6 \mathrm{~m}$ entre si, por lo que en cada corrida se ocuparon 7 separadores.

El programa de secado se efectuó con dos repeticiones y en cada una se procedió a identificar 60 piezas, 10 en cada paquete, escogiendo una tabla testigo por paquete que permitiera realizar los controles de contenido de humedad promedio, distribución de humedad y tensiones mediante la extracción de probetas. 
Tanto en estas piezas testigo (6) como en el resto de las piezas (54) se procedió a identificar el tipo de corte (floreado, cuarteado o mixto), a medir el ancho y el espesor con el fin de poder realizar el posterior cálculo de contracción (tanto en sentido tangencial, radial y volumétrico) y a identificar las posibles manchas, nudos y grietas que pudieran tener.

Como apoyo a los controles de humedad promedio realizados a través de probetas, se utilizó un xilohigrómetro que permitió llevar a cabo controles más seguidos de la humedad de la madera a medida que transcurría el proceso de secado.

El volumen de madera ingresado a la cámara para efectuar el programa de secado fue de 437,06 pulgadas (563 piezas).

Programa de Secado Tentativo

Tanto en la primera como en la segunda repetición se utilizó el mismo programa de secado convencional, el cual se confeccionó en base al contenido de humedad inicial de la madera.

Las temperaturas aplicadas en cada etapa se presentan en el Cuadro $N^{0} 1$. 


\section{Cuadro $N^{\bullet} 1$}

TEMPERATURAS APLICADAS POR ETAPA

\begin{tabular}{|c|c|c|c|}
\hline Tiempo & \multicolumn{2}{|c|}{ Temperatura } & \multirow{2}{*}{$\begin{array}{c}\text { Diferencia } \\
\text { Psicrométrica } \\
\left({ }^{\circ} \mathrm{C}\right)\end{array}$} \\
\hline (h) & $\begin{array}{l}\text { Seca } \\
\left({ }^{\circ} \mathrm{C}\right)\end{array}$ & $\begin{array}{l}\text { Húmeda } \\
\left({ }^{\circ} \mathrm{C}\right)\end{array}$ & \\
\hline $\begin{array}{c}\text { Calentamiento } \\
0,0 \\
1,5 \\
3,0\end{array}$ & $\begin{array}{l}25 \\
34 \\
42\end{array}$ & $\begin{array}{l}24 \\
32 \\
40\end{array}$ & $\begin{array}{l}1 \\
2 \\
2\end{array}$ \\
\hline $\begin{array}{c}\text { Vaporizado } \\
0,0 \\
2,5 \\
5,0 \\
\end{array}$ & $\begin{array}{l}42 \\
42 \\
42 \\
\end{array}$ & $\begin{array}{l}40 \\
40 \\
40\end{array}$ & $\begin{array}{l}2 \\
2 \\
2 \\
\end{array}$ \\
\hline Secado & $\begin{array}{l}42 \\
46 \\
47 \\
50 \\
52 \\
55 \\
58 \\
60 \\
65\end{array}$ & $\begin{array}{l}40 \\
44 \\
44 \\
44 \\
44 \\
47 \\
49 \\
51 \\
51\end{array}$ & $\begin{array}{c}2 \\
2 \\
3 \\
6 \\
8 \\
8 \\
9 \\
9 \\
14\end{array}$ \\
\hline
\end{tabular}

En ambas repeticiones del programa se pretende alcanzar un contenido de humedad final de 12 a $14 \%$.

\section{Controles Antes del Proceso de Secado}

- Determinación del contenido de humedad inicial: Esta medición se realizó en probetas extraídas de las 6 piezas testigos. El contenido de humedad inicial se determinó de acuerdo con las especificaciones de la norma NCh $176 / 3$ (1983).

- Paralelo a la extracción de las probetas se procedió a medir el contenido de humedad de las tablas testigo con xilohigrómetro. 
- Marcación de puntos para determinación de contracción : Esta marcación se efectuó tanto en el sentido tangencial como radial de las tablas, es decir se midió tanto el ancho como el espesor de las piezas elegidas (60).

- Identificación y observación de defectos: Previo a la formación de los paquetes se llevó a cabo la identificación visual de defectos, tales como grietas, nudos y manchas, los que fueron marcados con un lápiz de tinta indeleble en las 60 piezas escogidas.

\section{Controles Durante el Proceso de Secado}

- Determinación del contenido de humedad promedio : Estos se llevó a cabo de acuerdo a lo descrito en el control anterior.

- Distribución de humedad : El control de la distribución de humedad se efectuó a través de la obtención de probetas estratificadas de las mismas piezas testigo (6) y se obtuvo de la misma forma que para las probetas de humedad promedio.

- Tensiones : La determinación de las tensiones que presentaba la madera a través del programa de secado, se realizó mediante la obtención de las "probetas de tenedor" las que se obtuvieron de las 6 piezas testigo (Hildebrand, 1964).

\section{Controles Posteriores al Proceso de Secado}

- Contenido de humedad final : Se determinó mediante la obtención de las probetas de humedad promedio de las piezas testigo, así como sobre la base de la determinación de la humedad utilizando el xilohigrómetro para el total de las piezas (60) elegidas en este estudio.

- Distribución de humedad : Este control se realizó de acuerdo al procedimiento descrito en control antes de proceso de secado.

- Tensiones: Su determinación se realizó de la misma forma descrita en el control antes del proceso de secado.

- Contracción : De acuerdo a los puntos marcados y medidos en la etapa anterior al proceso de secado, tanto en el plano tangencial como radial, en 
esta etapa se procedió a medirlos nuevamente después de finalizado el proceso de secado. En base a esto se realizó el cálculo de la contracción según lo especificado en la Norma Chilena 176/3 (INN, 1983).

- Análisis de defectos : Este análisis se efectuó considerando los siguientes defectos : grietas, alabeos y colapso. La evaluación de las piezas en base a estos defectos, permitió establecer posteriormente la calidad del proceso de secado.

Las características que determinan estos defectos así como la forma en que fueron evaluados, se describen a continuación :

Alabeos o Deformaciones: Toda distorsión en una pieza de madera que lleve a desviaciones en su plano original. Su causa está en las diferencias existentes entre las contracciones radial, tangencial y longitudinal (Juacida, Insunza. 1986).

De acuerdo a esta definición los alabeos considerados en este trabajo son los siguientes :

Acanaladura (Cup) : Curvatura que se desarrolla en sentido transversal a las fibras.

Arqueadura (Bow) : Curvatura que presenta una pieza en el sentido longitudinal de las fibras. La oposición de sus caras llega a ser cóncava o convexa.

Encorvadura (Spring) : Alabeo de los cantos en la dirección longitudinal. Es también llamada "curvatura de cantos".

Torcedura (Twist) : Alabeo helicoidal de la pieza en tomo a su eje longitudinal.

Los alabeos anteriormente mencionados se evaluaron en base a la escuadría de las piezas, cuyos valores máximos admisibles se fundamentaron en lo descrito por INN (1972) para piezas de 3,6 metros de largo.

En base al nivel de defectos es posible ordenar el grado en que se produce el alabeo según su cambio dimensional, para luego relacionarlo con el coeficiente de ponderación, lo que permite establecer tanto la intensidad como la media del defecto. 
Grietas : En base a la metodología utilizada por Bravo (1977) y Muñoz (1972) y que es descrita por INN (1972). Esta establece los siguientes grados de clasificación :

Coeficiente de ponderación
A $(0,0)$
$B(0,5)$
C $(2,0)$
D $(2,5)$

Descripción

Sin defecto, sin grieta

Leve, hasta 4 grietas finas de 1" de longitud.

Leve a intenso, hasta 4 grietas abiertas de 1" de longitud

Intenso, más de 5 grietas abiertas y largas

Colapso : Este defecto se evaluó a través de la inspección visual de las piezas y se procedió luego a utilizar la siguiente pauta de evaluación aplicada también por Bravo (1977) y Ramírez (1984).

Coeficiente de Ponderación

$\operatorname{SC}(0,0)$

$\mathrm{CL}(0,5)$

Cl $(1,5)$

\section{Descripción}

Sin colapso, la madera no presenta aplastamiento celular.

Colapso leve, se presenta aplastamiento celular en algunas zonas de la pieza.

Colapso intenso, la pieza está más de un $50 \%$ afectada por aplastamiento celular.

Calidad del Programa de Secado

Con respecto a la determinación de la calidad del secado para ambas repeticiones, se optó por el sistema empleado por Bravo (1977), el cual se basa en la cuantificación de defectos tales como grietas, alabeos y colapso, según 
las sumas de sus medias estadísticas. Se establece una ponderación en base a la adaptación de los cuatro índices ya mencionados, los cuales tomarán valores de acuerdo a la ausencia o mayor intensidad del defecto, según se muestra en el siguiente Cuadro $\mathrm{N}^{\circ} 2$.

\section{Cuadro $\mathrm{N}^{\circ} 2$}

ESCALA DE REFERENCIA PARA DETERMINAR LA CALIDAD DEL SECADO

\begin{tabular}{|l|c|c|}
\hline Calidad de secado & Indice & Condiciones del secado \\
\hline Excelente & 0,0 & Adecuadas \\
Muy buena & $<0,5$ & \\
Buena & 1,0 & Poco adecuadas \\
\hline Regular & 1,5 & \\
Menos que regular & 2,0 & Inadecuadas \\
\hline Defectuosa & 3,0 & \\
Mala & 5,0 & \\
Muy mala & $>5,0$ & \\
\hline
\end{tabular}

Fuente : Bravo, 1977

Análisis Estadístico

Se efectuó un análisis de varianza con un nivel de significación del $5 \%$ y un análisis de comparación múltiple de Bonferroni.

\section{PRESENTACION Y ANALISIS DE RESULTADOS}

En el programa de secado establecido se puso énfasis en que la rigurosidad de las temperaturas fuera aumentando gradualmente, poniendo especial cuidado al momento en que los contenidos de humedad de la madera fueran cercanos al punto de saturación de las fibras, con el fin de minimizar los riesgos de defectos que en esta etapa se producen. Desde este punto las diferencias 
psicrométricas de las temperaturas seca y húmeda se hicieron mayores adquiriendo de esta manera el programa de secado una mayor rigurosidad.

\section{Caracterización del Programa de Secado}

El programa de secado fue elaborado exclusivamente en base a la pérdida de humedad que experimenta la madera en el transcurso del proceso, con el apoyo de controles sucesivos de contenido de humedad tratando de establecer un programa sin cambios demasiado rigurosos (cambios de temperaturas), que pueden afectar fuertemente la calidad final de la madera.

Al analizar la evolución del programa, se puede destacar que al final del proceso de secado, el cual tuvo una duración de 96 horas, se logró alcanzar un contenido de humedad promedio de la madera de un $13.93 \%$ (Figura $N^{\circ} 1$ ).

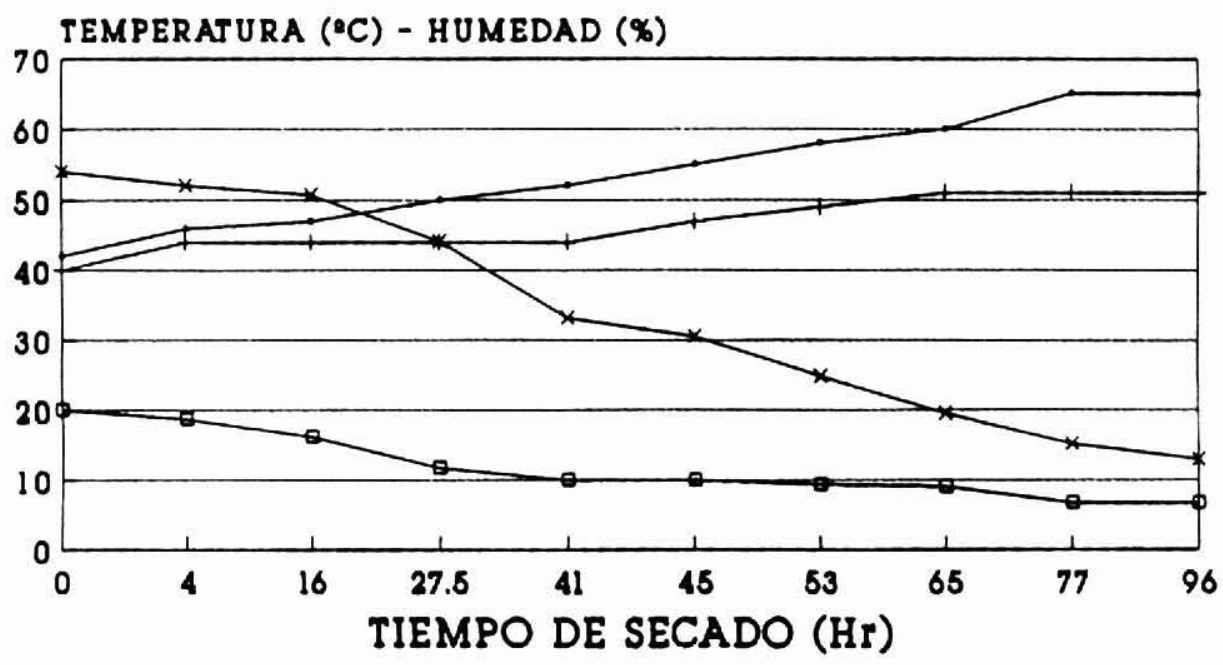

\footnotetext{
$\rightarrow$ TEMPERATURA SECA

$\rightarrow$ HOM. EQ. MADERA
}

十 TEMPERATURA BUMEDA

* GUMEDAD DE LA MADERA

Figura No 1. PLAN DE SECADO Y CURVA DE SECADO PARA LA ESPECIE TEPA 
Mediante el análisis de varianza se buscó la existencia de diferencias significativas de los contenidos de humedad promedio entre los paquetes, según la ubicación que estos presentaban dentro de la cámara de secado. También se evaluó la posible incidencia del tipo de corte en el contenido de humedad final de las piezas muestra.

Según este análisis se concluyó que el tipo de corte no afectó el contenido de humedad final de la madera ya que la probabilidad arrojada fue de 0,624 valor que se encuentra considerablemente fuera de la zona de significación.

En cambio en la ubicación de los paquetes, se evidencia que las diferencias en los contenidos de humedad de un paquete a otro no son atribuibles a causas aleatorias, sino que por el contrario, existen razones suficientes que conducen a afirmar que la distribución de los 6 paquetes de madera dentro de la cámara de secado estaría afectando el contenido de humedad obtenido al final del tiempo de secado $(p=0,0028)$.

Las diferencias en cuanto al contenido de humedad final según la ubicación de los paquetes, permite inferir que la causa radicaria en la humedad inicial o en que el flujo del aire dentro de la cámara no es homogéneo. De acuerdo a esto último y en base a las mediciones de velocidad del aire realizadas en la cámara, se puede asumir que existe una aireación que en promedio presenta diferencias, de una dirección con respecto a la otra (norte-sur), que no permitirian homogeneizar la humedad final entre los paquetes.

\section{Contracción}

Debido a la importancia que presenta la escuadría final de la madera después del secado, se procedió a medir la contracción experimentada por las piezas escogidas como muestra, las cuales fueron medidas antes de iniciar el proceso de secado y que además fueron identificadas según el tipo de corte que presentaban (floreado, cuarteado o mixto).

De acuerdo a esto último, los valores de contracción radial y tangencial promedio por paquete se refieren a piezas que presentaban corte floreado 0 
cuarteado y con respecto al promedio de contracción volumétrica, además de los cortes mencionados, se incluye el corte mixto en el promedio.

\section{Cuadro $\mathrm{N}^{\circ} 3$}

CONTRACCION PROMEDIO DE LA MADERA SOMETIDA A SECADO

\begin{tabular}{|c|c|c|c|}
\hline \multirow{2}{*}{$N^{\circ}$ Paquete } & \multicolumn{3}{|c|}{ Contracción (\%) } \\
\cline { 2 - 4 } & Radial & Tangencial & Volumétrica \\
\hline 1 & 2,41 & 4,80 & 7,21 \\
2 & 3,80 & 4,66 & 8,46 \\
3 & 3,32 & 3,85 & 7,17 \\
4 & 3,32 & 4,24 & 7,56 \\
5 & 3,81 & 4,43 & 8,24 \\
6 & 3,64 & 6,19 & 9,83 \\
\hline
\end{tabular}

En los valores de contracción promedio entregados en el Cuadro $\mathrm{N}^{\circ} 3$ se aprecia que en todos los paquetes existe un mayor porcentaje de contracción tangencial respecto a la radial, lo cual permite establecer que esta especie presenta en promedio una mayor contracción tangencial. Esto coincide con los resultados obtenidos por Inzunza (1973), aunque en su trabajo los valores de contracción tangencial encontrados fueron superiores.

En relación al tipo de corte de las piezas muestra, se encontró que aquellas de corte floreado (30) tuvieron un $8.38 \%$ de contracción volumétrica y las de corte cuarteado (14) y mixto (16) contracciones de $8,01 \%$ y $7,91 \%$. respectivamente (Figura $\mathrm{N}^{\circ} 2$ ). 


\section{BIBLIOTECA

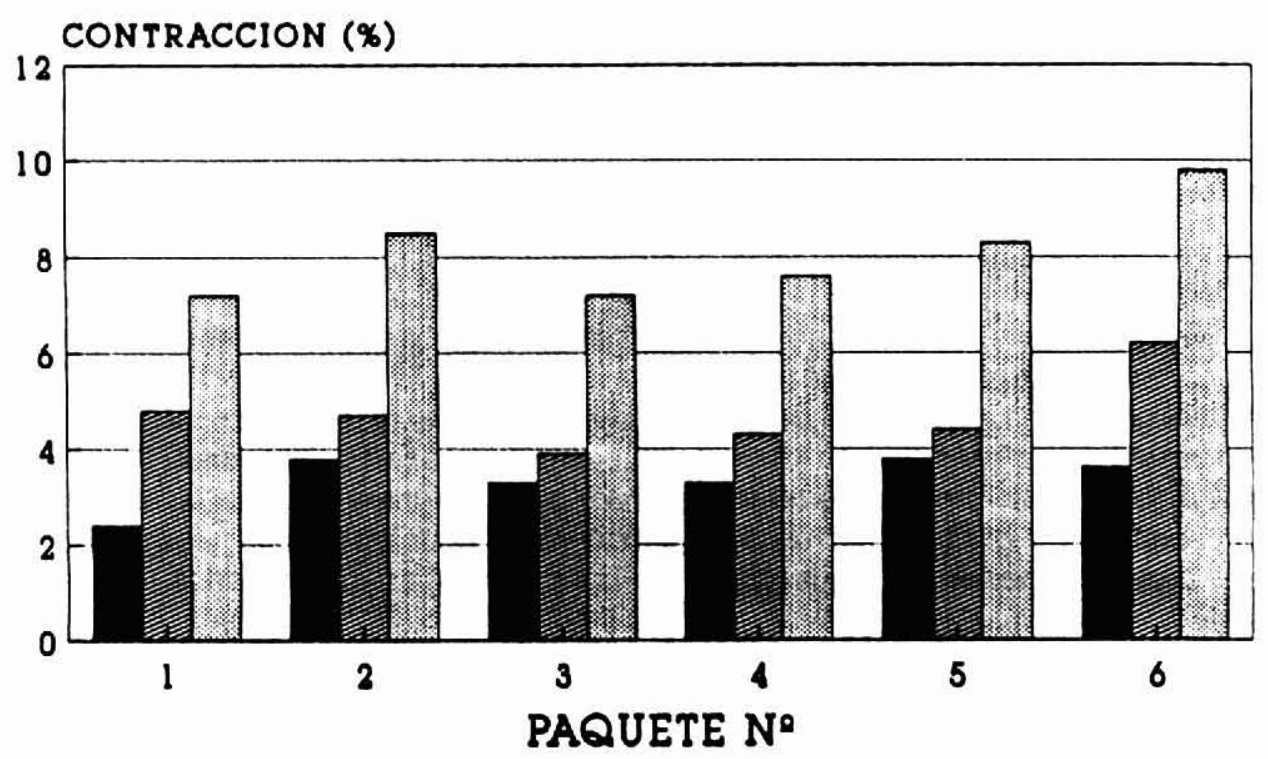

Radial W Tangenclal Wolumétrica

Figura $N^{\circ}$ 2. CONTRACCION DE LA MADERA SOMETIDA A SECADO

\section{Calidad de Secado}

La determinación de la calidad del secado se llevó a cabo sobre la base de los controles realizados a las piezas muestra al final del proceso de secado, con el fin de identificar y medir los defectos que ellas presentaban y así poder concluir el nivel de calidad observado.

Colapso : No se observó presencia de colapso en la evaluación final de las piezas.

Alabeos : La deformación que se observó en un mayor número de piezas correspondió a la acanaladura, la cual se presentó en un total de 20 piezas 
muestra lo que representa el $37,03 \%$ de las piezas muestreadas para este tipo de defecto. El mayor número de piezas afectadas por este tipo de alabeo correspondió a aquellas con corte mixto (14,81\%), un $12,96 \%$ a piezas con corte cuarteado y un $9.26 \%$ correspondió a piezas floreadas. Con respecto a esto, y debido a que por lo general la acanaladura se encuentra asociada a piezas con corte floreado, existe la posibilidad que al realizar la clasificación del tipo de corte, existan piezas que fueron consideradas con corte mixto y que realmente pudieron tener un mayor porcentaje de corte tangencial respecto al radial.

Sin embargo, la deformación que se presentó con mayor intensidad fue la encorvadura cuya media estadística fue de 0,315 y la acanaladura con 0,241. Si se considera el tipo de corte, es posible señalar que la intensidad de la encorvadura se presentó en mayor porcentaje en piezas cuarteadas $(14,81 \%)$, luego en piezas con corte mixto $(3,7 \%)$ y finalmente floreadas $(1,86 \%)$, presentándose el mayor número de piezas con encorvadura vinculadas al tipo de corte cuarteado, al igual que en las referencias presentadas de otros estudios.

Con respecto a los defectos arqueadura y torcedura, la mayor parte de las piezas no presentaron este tipo de defectos $(87,03 \%$ y $83,33 \%$, respectivamente) y en consecuencia sus medias estadísticas, que representan la intensidad del defecto, fueron relativamente bajas, siendo la torcedura la que menos influyó.

Grietas : de acuerdo a las observaciones realizadas al final del secado, se constató que el número de piezas afectadas por grietas fue mucho menor a las que presentaban alabeos.

Se identificó un mayor número de piezas con presencia de grietas en las caras existiendo un $22,2 \%$ de agrietamiento leve y un $7,41 \%$ leve - intenso. En cambio las grietas en las puntas afectaron un menor número de piezas, identificándose un $11,11 \%, 3,55 \%$ y $1,85 \%$ de grietas con intensidades leve, leve-intenso e intenso, respectivamente.

La media estadística total corresponde a 1,305 lo que significa, al relacionarlo con la pauta establecida anteriormente, que la calidad del proceso de secado fue de regular a buena.

De acuerdo a los contenidos de humedad iniciales y finales obtenidos y al tiempo de secado empleado, es importante determinar la velocidad media de 
secado mediante la fórmula usada por Kollmann (1961), Ladell (1957) y Bravo (1977).

$$
V(\% h)=\frac{H i-H f}{h}
$$

Donde :

$$
\begin{array}{ll}
\mathrm{V}= & \begin{array}{l}
\text { Velocidad media de secado, la cual expresa la pérdida de } \\
\text { humedad }(\%) \text { que experimenta la madera por hora de } \\
\text { secado. }
\end{array} \\
\mathrm{Hi} & =\quad \text { Humedad inicial promedio (\%) de la carga } \\
\mathrm{Hf} & =\quad \text { Humedad final promedio (\%) de la carga } \\
\mathrm{h} & =\quad \text { Tiempo total de secado }(\mathrm{h})
\end{array}
$$
humedad (\%) que experimenta la madera por hora de

Los valores de velocidad media de secado obtenidos utilizando la fórmula presentada anteriormente, revelan que esta pérdida hasta el punto de saturación de fibras fue de $0,51 \% / \mathrm{h}$ y posterior a este punto la pérdida de humedad fue de $0,33 \% / \mathrm{h}$. 


\section{CONCLUSIONES}

El programa de secado establecido par la especie Laurelia philippiana, en base al contenido de humedad de la madera, tuvo un tiempo de duración de 96 horas, alcanzando un contenido de humedad final promedio de $12,93 \%$.

La calidad del secado, se considera de regular a buena al medir la efectividad a través de la media estadistica total, el valor obtenido fue 1,305.

El defecto con mayor incidencia en la calidad del secado fue el alabeo y en segundo lugar se presentaron las grietas. Los valores obtenidos son 63,83 y 35,39 $\%$, respectivamente.

La encorvadura tuvo un porcentaje de participación de un $24,14 \%$, afectando al $14,81 \%$ de las piezas con corte mixto, seguida por el defecto acanaladura cuya participación fue de un $18,4 \%$, afectando a un $37,03 \%$ del total de piezas. Las más afectadas son aquellas de corte mixto $(14,81 \%)$. Es decir, la acanaladura afectó un mayor número de piezas y la encorvadura fue el defecto más intenso.

Las grietas en las caras tuvieron mayor participación (19,85\%) respecto a las presentadas en las puntas $(16,32 \%)$.

La contracción tangencial en promedio fue mayor que la radial, así como la mayor contracción volumétrica promedio se alcanzó en piezas con corte floreado.

La presencia de grietas por lo general se vio asociada a piezas que presentaban una mancha longitudinal de color negro, lo cual indicaria que estas piezas son más propensas a este tipo de defecto y podrían implicar perjuicios en la propiedades de la madera. 


\section{REFERENCIAS BIBLIOGRAFICAS}

Bahamondez, R., 1976. Proposición de un Programa de Secado Artificial para Madera de Coigüe de Magallanes (Nothofagus betuloides (Mirb). Oerst) de hasta $1 \quad 1 / 2^{\prime \prime}$ de Espesor Previa Aplicación y Análisis de Tres Programas Tentativos. Tesis Ing. Forestal, Facultad Cs. Forestales. Universidad Austral de Chile. Valdivia. 11 p.

Bluhm, E., Kauman, W., Melo, R., Neira, M., 1965. El Colapso en la Madera y su Reacondicionamiento. Ensayos de Recuperación de Colapso en Madera de Eucalipto (Eucalyptus globulus) Crecido en Chile. Informe Técnico $\mathrm{N}^{\circ} 22$. Instituto Forestal. $21 \mathrm{p}$.

Bravo, A., 1977. Comportamiento de Pino Insigne (Pinus radiata D. Don) Bajo Dos Condiciones de Secado por Vapor Recalentado. Tesis Ing. Forestal, Facultad Cs. Forestales. Universidad Austral de Chile. Valdivia. $77 \mathrm{p}$.

Corporación Nacional Forestal, 1986. Proyecto de Incentivos al Manejo del Bosque Nativo. Documento de Trabajo $N^{\circ}$ 79. 213 p.

Cuevas, E., 1969. Incidencia de los Fenómenos de Contracción y Colapso en el Secado de Algunas Maderas Comerciales en Chile. Cuarta Reunión sobre Investigación en Productos Forestales. Instituto Forestal. Santiago, Chile. pp: 145-154.

Gutierrez, M., 1985. Estudio de Secado Industrial en Cámara, de Madera Aserrada de Tepa. Resumen 4. Escuela de Ciencias Forestales. Universidad de Chile. Santiago, Chile. pp: $31-41$.

Hildebrand, R., 1964. El Secado de Madera Aserrada. Comercial Sagrera S.A. Barcelona, España. 159 p.

Instituto Nacional de Normalización Nch 993., 1972. EOf72. Madera. Procedimientos y Criterios de Evaluación para Clasificación. Santiago, Chile. 18p.

Instituto Nacional de Normalización. NCh 176/1 cR83., 1983. Madera. Determinación de Humedad. Santiago, Chile. $18 \mathrm{p}$.

Instituto Nacional de Normalización. NCh 176/3 cR83., 1983. Madera. Determinación de Contracción Radial y Tangencial. Santiago, Chile. 9 p. 
Inzunza, L., 1973. Determinación de las Contracciones de las Especies de Mayor Importancia Comercial en Chile. Universidad Austral de Chile. Instituto de Tecnologia de la madera. Valdivia, Chile. $31 \mathrm{p}$.

Juacida, R. y Inzunza, L., 1986. Pautas de Control de Calidad para Madera Secada Artificialmente. Universidad Austral de Chile. Facultad Cs. Forestales. Publicación Docente $\mathrm{N}^{\circ} 21.27 p$.

Kauman, W., Mittak, G., 1965. Ensayos de Secado en Coigũe (Nothofagus Dombeyi). Informe Técnico $\mathrm{N}^{\circ} 25$. Instituto Forestal. Santiago, Chile. 25p.

Kollmann, F., 1961. High-Temperature Drying: Research, Application and Experience in Germany. For. Prod. Journal. 11(11): 508-515.

Muñoz, R., 1972. Ensayo con Tres Programas de Secado Artificial en Cinco Especies Nativas Latifoliadas. Tesis Ing. Forestal. Facultad de Cs. Forestales. Universidad Austral de Chile. Valdivia. $112 \mathrm{p}$.

Peredo, M. Inzunza, L., 1988. Secado de Maderas. Universidad Austral de Chile. Facultad Cs. Forestales. Centro Experimental Forestal. Unidad de Capacitación. Valdivia, Chile $75 \mathrm{p}$.

Pérez, V., 1983. Manual de Propiedades Físicas y Mecánicas de Maderas Chilenas. Investigación y Desarrollo Forestal. Documento de Trabajo $N^{\circ} 47$. Santiago, Chile, 451p.

Ramírez, J., 1984. Comportamiento de Pino Oregón, Pseudotsuga menziesii (Mirb.) Franco, Frente al Secado Artificial. Tesis Ing. Forestal, Facultad Cs. Forestales. Universidad Austral de Chile. Valdivia. $87 \mathrm{p}$.

Rosende, R. y Castillo, H., 1973. Contracción, Colapso y Juego de las Maderas Chilenas. Instituto Forestal. Boletín Informativo $N^{\circ} 27$. Santiago Chile, 39 p.

Salgado, S., 1974. Aplicación y Análisis de Tres Programas de Secado Artificial en Madera de Lenga, Nothofagus dombeyi (Kasser)). Tesis Ing. Forestal. Facultad de Cs. Forestales. Universidad Austral de Chile. Valdivia, $49 \mathrm{p}$.

Stevens, M.; Mech, A. y Pratt, G., 1954. Seasoning Wood at High Temperatures. Timber Technology and Machine Woodworking. 62(12) : 599-602.

Wright, G., 1956. The Seasoning of the Eucalyptus. Commonwealth Scientific and Industrial Research Organization. Div. of. For. Prod. 12 p. 